\title{
On orbits of unipotent flows on homogeneous spaces
}

\author{
S. G. DAN I \\ School of Mathematics, Tata Institute of Fundamental Research, Homi Bhabha Road, \\ Bombay 400005, India
}

(Received 14 February 1983)

\begin{abstract}
Let $G$ be a connected Lie group and let $\Gamma$ be a lattice in $G$ (not necessarily co-compact). We show that if $\left(u_{i}\right)$ is a unipotent one-parameter subgroup of $G$ then every ergodic invariant (locally finite) measure of the action of $\left(u_{t}\right)$ on $G / \Gamma$ is finite. For 'arithmetic lattices' this was proved in [2]. The present generalization is obtained by studying the 'frequency of visiting compact subsets' for unbounded orbits of such flows in the special case where $G$ is a connected semi-simple Lie group of R-rank 1 and $\Gamma$ is any (not necessarily arithmetic) lattice in $G$.
\end{abstract}

\section{Introduction}

Let $G$ be a connected Lie group and $\Gamma$ be a lattice in $G$; that is, $G / \Gamma$ admits a finite $G$-invariant (Borel) measure. Let $\left(u_{t}\right)_{t \in \mathbf{R}}$ be a one-parameter subgroup consisting of unipotent elements (that is, each $\operatorname{Ad~} u_{t}$ is a unipotent linear transformation of the Lie algebra of $G$ ). The action of such a one-parameter group on $G / \Gamma$ (on the left) is dubbed a unipotent flow.

In the particular case when $G=\operatorname{SL}(n, \mathbb{R})$ and $\Gamma=\operatorname{SL}(n, \mathbb{Z})$ it was proved by Margulis [6] that for any $x \in G / \Gamma$ the positive semi-orbit $\left\{u_{t} x \mid t \geq 0\right\}$ of a unipotent flow does not tend to infinity; that is, there exists a compact set $K$, depending on $x$, such that the set

$$
E_{K}(x)=\left\{t \geq 0 \mid u_{t} x \in K\right\}
$$

is unbounded. In [2] the present author strengthened this assertion by proving that for a suitable compact set $K$ the set $E_{K}(x)$ is of positive density; that is,

$$
\liminf _{T \rightarrow \infty} \frac{1}{T} l\left(E_{K}(x) \cap[0, T]\right)>0,
$$

where $l$ is the usual Haar measure on $\mathbb{R}$. Further as an application of this it was deduced that every locally finite ergodic invariant measure of a unipotent flow, and more generally of the action of any subgroup consisting only of unipotent elements, is finite.

Using certain standard facts about arithmetic subgroups the above results can easily be generalized to the situation where $G$ is any connected Lie group and $\Gamma$ is an arithmetic lattice in $G$ (cf. [2] for details). In [3], where the above finiteness assertion was used crucially in the classification of invariant measures of maximal 
horospherical flows, the author raised the question of whether the results hold also for non-arithmetic lattices. The purpose of this note is to show that that is indeed the case (cf. theorem 4.1).

As a knowledgable reader would recognize, in view of Margulis's arithmeticity theorem the above question reduces to its special case where $G$ is a simple Lie group of $\mathbb{R}$-rank 1 . In the present article we shall in fact prove that for any lattice in a simple Lie group of $\mathbb{R}$-rank 1 we can demonstrate an even stronger version of (0.1), where the right hand side is replaced by $1-\varepsilon, \varepsilon>0$ and that the compact set could be chosen to be the same for all unbounded orbits. There are of course, bounded orbits lying outside any given compact set. For these $(0.1)$ holds anyway with the closure as the compact set in question. Specifically we have the following:

(0.2) TheOREM. Let $G$ be a connected semisimple Lie group of $\mathbb{R}$-rank 1 and $\Gamma$ be a lattice in $G$. Let $\left(u_{t}\right)_{t \in \mathbb{R}}$ be a unipotent one-parameter subgroup of $G$. Then given $\varepsilon>0$ there exists a compact subset $C$ of $G / \Gamma$ such that for any $x \in G / \Gamma$ whose orbit $\left\{u_{t} x \mid t \in \mathbb{R}\right\}$ is not bounded (i.e. relatively compact) in $G / \Gamma$ we have

$$
\liminf _{T \rightarrow \infty} \frac{1}{T} \int_{0}^{T} \chi_{C}\left(u_{t} x\right) d t>1-\varepsilon,
$$

$\chi_{C}$ being the characteristic function of $C$ on $G / \Gamma$.

The method of proof is fundamentally different and in some ways more natural than in [2]. However unfortunately it seems to work only for $\mathbb{R}$-rank 1 groups. It may be noted that the method is reminiscent of [4], where it was proved that for $G=\operatorname{SL}(2, \mathbb{R})$ any non-periodic orbit of a unipotent flow as above is in fact uniformly distributed with respect to the $G$-invariant measure.

Combining theorem 0.2 with the results of [2] and arguing as in the latter it is easy to deduce the following, (cf. theorem 4.3).

(0.3) Theorem. Let $G$ be a connected Lie group and let $\Gamma$ be a lattice in $G$. Let $U$ be a subgroup of $G$ consisting only of unipotent elements. Then any locally finite ergodic $U$-invariant measure on $G / \Gamma$ is finite.

Acknowledgement. It is a pleasure to thank John Smillie, some of whose ideas, learnt by the author while collaborating on [4] turned out to be very useful in proving theorem 0.2 .

\section{Preliminaries and notation}

Let $G$ be a connected semisimple Lie group of $\mathbb{R}$-rank 1 ; that is, any maximal vector subgroup whose adjoint action on the Lie algebra of $G$ is (simultaneously) diagonalizable over $\mathbb{R}$ is one-dimensional. Let $g$ be the Lie algebra of $G$ and let $A$ be a one-parameter subgroup whose adjoint action on $g$ is diagonalizable over $\mathbb{R}$. Let $Z$ be the centralizer of $A$, the subgroup consisting of all elements which commute with each element of $A$. Let be the Lie subalgebra associated to $Z$. There exists a unique character $\alpha: A \rightarrow \mathbb{R}^{+}$such that

$$
g=g_{-2}+g_{-1}+g_{1}+g_{2}
$$


where

$$
g_{i}=\left\{X \in g \mid(\operatorname{Ad} a) X=\alpha(a)^{i} X\right\}, \quad i= \pm 1, \pm 2,
$$

and $g_{ \pm 1}$ are positive dimensional. We note that $g_{ \pm 2}$ may be trivial. For any $s>0$ put

$$
A_{s}=\{a \in A \mid \alpha(a)<s\} \text {. }
$$

Let $N$ be the analytic subgroup corresponding to $g_{1}+g_{2}$ (the latter is indeed a Lie subalgebra of $g$ ). It then follows easily that every element of $Z$ normalizes $N$. Put $P=Z N$. Then $P$ is a minimal parabolic subgroup of $G$. In what follows we shall also fix a maximal compact subgroup $K$ of $G$. For any $s>0$ and any compact subset $\eta$ of $N$ let

$$
\Omega(s, \eta)=K A_{s} \eta
$$

Now let $\Gamma$ be a non-uniform lattice in $G$; that is, $\Gamma$ is a discrete subgroup such that $G / \Gamma$ is non-compact but admits a finite $G$-invariant measure. We need the following result due to Garland and Raghunathan (cf. [5, theorems 0.6 and 0.7 ).

(1.1) ThEOREM. Let the notation be as above. There exists $s_{0}>0$, a compact subset $\eta_{0}$ of $N$ and a finite subset $\Sigma$ of $G$ such that the following assertions hold:

(i) $G=\Omega\left(s_{0}, \eta_{0}\right) \Sigma \Gamma$.

(ii) For all $\sigma \in \Sigma, \Gamma \cap \sigma^{-1} N \sigma$ is a (cocompact) lattice in $\sigma^{-1} N \sigma$.

(iii) For all compact subsets $\eta$ of $N$ the set

$$
\left\{\gamma \in \Gamma \mid \Omega\left(s_{0}, \eta\right) \Sigma \gamma \cap \Omega\left(s_{0}, \eta\right) \neq \varnothing\right\}
$$

is finite.

(iv) Given a compact subset $\eta$ of $N$ containing $\eta_{0}$, there exists $s_{1} \in\left(0, s_{0}\right)$ such that whenever $\sigma, \tau \in \Sigma$ are such that $\Omega\left(s_{0}, \eta\right) \sigma \gamma \cap \Omega\left(s_{1}, \eta\right) \tau$ is non-empty for some $\gamma$ then $\sigma=\tau$ and $\sigma \gamma \sigma^{-1} \in(K \cap Z) \cdot N \subset P$.

In view of assertion (ii) above, there exists a compact subset $\eta$ of $N$ containing $\eta_{0}$ such that $\eta \sigma\left(\sigma^{-1} N \sigma \cap \Gamma\right)=N \sigma$ for all $\sigma \in \Sigma$. In what follows we shall fix such a set $\eta$ and $s_{1} \in\left(0, s_{0}\right)$ so that assertion (iv) in the theorem is satisfied for the set $\eta$. For $\sigma \in \Sigma$ and $s<s_{0}$ put

$$
X(\sigma, s)=\Omega(s, \eta) \sigma \Gamma / \Gamma .
$$

In view of assertion (i) in the theorem, for any $s<s_{0}$ the complement of $\bigcup_{\sigma \in \Sigma} X(\sigma, s)$ in $G / \Gamma$ is compact. Since $\Sigma$ is finite this means that in order to prove theorem 0.2 it is enough to prove the following:

(1.2) Proposition. Let the notation be as above. Let $\left(u_{t}\right)$ be a unipotent one-parameter subgroup of $G$. Let $\sigma \in \Sigma$ and $\varepsilon>0$ be given. Then there exists $s>0$ such that for any $x \in G / \Gamma$ either $\left\{u_{t} x \mid t \in \mathbb{R}\right\}$ is bounded in $G / \Gamma$ or

$$
\limsup _{T \rightarrow \infty} \frac{1}{T} \int_{0}^{T} \chi_{s}\left(u_{t} x\right) d t \leq \varepsilon,
$$

where $\chi_{s}$ is the characteristic function of $X(\sigma, s)$ in $G / \Gamma$.

The proof of the proposition will be completed in $\S 3$. As is now justified, along with the data as in theorem 0.2 we shall henceforth assume $\sigma \in \Sigma$ to be given. 
Now let $L$ be the connected component of the identity in $\sigma^{-1}(K \cap Z) \cdot N \sigma$. Then $L$ is a normal subgroup of $\sigma^{-1} P \sigma$ of codimension 1 . In view of assertion (ii) of theorem 1.1, $L \cap \Gamma$ is a lattice in $L$.

(1.3) Remark. For any $s>0, K A_{s} \eta \sigma(L \cap \Gamma)=K A_{s} \sigma L$.

Proof. Since $\sigma^{-1} \eta \sigma \subset \sigma^{-1} N \sigma \subset L$ we get

$$
K A_{s} \eta \sigma(L \cap \Gamma) \subset K A_{s} \sigma L .
$$

On the other hand $\sigma L \sigma^{-1} \subset(K \cap Z) \cdot N$ and $N \sigma \subset \eta \sigma(L \cap \Gamma)$, the latter by choice of $\eta$. Thus

$$
K A_{s} \sigma L \subset K A_{s}(K \cap Z) N \sigma=K A_{s} N \sigma \subset K A_{s} \eta \sigma(L \cap \Gamma)
$$

\section{A representation}

We now define a representation $\rho$ of $G$ as follows. Let $l$ be the dimension of $L$ and let $V=\Lambda^{\prime} g$, the $l$ th exterior power of $g$ (as a vector space). The subalgebra associated to $L$ determines a one-dimensional subspace of $V$. Let $v$ be any non-zero vector in this subspace. Let $\rho$ be the $l$ th exterior power of the adjoint representation of $G$ on $g$. Since $L$ is normal in $\sigma^{-1} P \sigma$ there exists a homomorphism $\theta: \sigma^{-1} P \sigma \rightarrow \mathbb{R}^{*}$ such that $\rho(x) v=\theta(x) v$ for all $x \in \sigma^{-1} P \sigma$. It is easy to verify that $\theta(x)=1$ for all $x \in L$. Evidently the vector $\rho(\sigma) v$ is a highest weight vector of the representation $\rho$ with respect to the minimal parabolic subgroup $P$, and the corresponding weight character on $A$ is given by $a \mapsto \theta\left(\sigma^{-1} a \sigma\right)$. Therefore there exists $q>0$ (actually a half-integer) such that for all $a \in A$,

$$
\theta\left(\sigma^{-1} a \sigma\right)=\alpha(a)^{q} .
$$

In what follows let $\langle$,$\rangle be a \rho(K)$-invariant inner product on $V$ and let $\|\cdot\|$ be the corresponding norm. For any $\delta>0$ let $B_{\delta}$ be the open ball (with respect to the norm $\|\cdot\|)$ in $V$ with centre at 0 and radius $\delta$. Also for any $s>0$ let

$$
\delta(s)=s^{q}\|\rho(\sigma) v\|
$$

where $q>0$ is as in (2.1). In particular $\delta$ is a monotonically increasing function on $\mathbb{R}^{+}$and $\delta(s) \rightarrow 0$ as $s \rightarrow 0$.

(2.2) Lemma. For any $s>0$,

$$
K A_{s} \sigma L=\left\{g \in G \mid \rho(g) v \in B_{\delta(s)}\right\} .
$$

Proof. Let $g=k a \sigma \xi$, where $k \in K, a \in A_{s}$ and $\xi \in L$. Then

$$
\begin{aligned}
\|\rho(g) v\| & =\|\rho(k) \rho(a) \rho(\sigma) \rho(\xi) v\|=\|\rho(a) \rho(\sigma) v\| \\
& =\theta\left(\sigma^{-1} a \sigma\right)\|\rho(\sigma) v\|=\alpha(a)^{q}\|\rho(\sigma) v\| \\
& <s^{q}\|\rho(\sigma) v\|=\delta(s) .
\end{aligned}
$$

Therefore $\rho(g) v \in B_{\delta(s)}$. Conversely let $g \in G$ be such that $\rho(g) v \in B_{\delta(s)}$. By the Iwasawa decomposition there exists $k \in K, a \in A$ and $n \in N$ such that $g \sigma^{-1}=k a n$. Since $N \subset \sigma L \sigma^{-1}, n$ may be expressed as $\sigma \xi \sigma^{-1}$, where $\xi \in L$. Thus $g=k a \sigma \xi$, where $k \in K, a \in A$ and $\xi \in L$. Since $\rho(g) v \in B_{\delta(s)}$ and $B_{\delta(s)}$ is $\rho(K)$-invariant using (2.1) 
we deduce that

Therefore

$$
\begin{aligned}
\alpha(a)^{q} \rho(\sigma) v & =\theta\left(\sigma^{-1} a \sigma\right) \rho(\sigma) v=\rho(a) \rho(\sigma) v \\
& =\rho(a) \rho(\sigma) \rho(\xi) v \in B_{\delta(s)}
\end{aligned}
$$

$$
\alpha(a)^{q}\|\rho(\sigma) v\|<\delta(s)=s^{q}\|\rho(\sigma) v\| .
$$

Hence $\alpha(a)<s$, or in other words $a \in A_{s}$. Thus $g=k a \sigma \xi \in K A_{s} \sigma L$, which proves the lemma.

(2.3) Corollary. Let $g \in G$. Then $g \Gamma \in X(\sigma, s)$ if and only if there exists $\gamma \in \Gamma$ such that $\rho(g \gamma) v \in B_{\delta}$, where $\delta=\delta(s)$. Furthermore, if $s \leq s_{1}$ and $\gamma_{1}$ and $\gamma_{2}$ in $\Gamma$ are such that $\rho\left(g \gamma_{i}\right) v \in B_{\delta}, i=1,2$ then

$$
\rho\left(g \gamma_{1}\right) v= \pm \rho\left(g \gamma_{2}\right) v .
$$

Proof. If $g \Gamma \in X(\sigma, s)$ then by the definition of $X(\sigma, s)$ there exists $\gamma \in \Gamma$ such that

$$
\mathrm{g} \gamma \in \boldsymbol{\Omega}(s, \eta) \sigma=K A_{s} \eta \sigma \subset K A_{s} \sigma L .
$$

Then by lemma 2.2, $\rho(g \gamma) v \in B_{\delta}$. Conversely suppose that $\rho(g \gamma) v \in B_{\delta}$ for some $\gamma \in \Gamma$. Then by lemma 2.2 and remark 1.3, $g \gamma \in K A_{s} \sigma L \subset K A_{s} \eta \sigma \Gamma$. Hence $g \Gamma \in X(\sigma, s)$.

Next suppose $s \leq s_{1}$ and $\gamma_{1}, \gamma_{2} \in \Gamma$ are such that $\rho\left(g \gamma_{i}\right) v \in B_{\delta}, i=1,2$. Then as above

$$
g \gamma_{i} \in K A_{s} \sigma L \subset K A_{s} \eta \sigma(L \cap \Gamma) \text {. }
$$

That is, there exist $\pi_{1}, \pi_{2} \in L \cap \Gamma$ such that

$$
g \in \Omega(s, \eta) \sigma \pi_{1} \gamma_{1}^{-1} \cap \Omega(s, \eta) \sigma \pi_{2} \gamma_{2}^{-1} .
$$

By assertion (iv) in theorem 1.1 this implies that

$$
\gamma_{1}^{-1} \gamma_{2} \in \sigma^{-1}(K \cap Z) \cdot N \sigma \text {. }
$$

But $L$ is of finite index in $\sigma^{-1}(K \cap Z) \cdot N \sigma$. Hence $\left(\gamma_{1}^{-1} \gamma_{2}\right)^{p} \in L$, where $p$ is the index in question. By an earlier remark we get that

$$
\rho\left(\left(\gamma_{1}^{-1} \gamma_{2}\right)^{p}\right) v=\theta\left(\gamma_{1}^{-1} \gamma_{2}\right)^{p} v=v
$$

which implies that $\theta\left(\gamma_{1}\right)= \pm \theta\left(\gamma_{2}\right)$ and in turn that

$$
\rho\left(g \gamma_{1}\right) v= \pm \rho\left(g \gamma_{2}\right) v .
$$

As a consequence of corollary 2.3 there exists a well-defined function $\varphi: X\left(\sigma, s_{1}\right) \rightarrow \mathbb{R}^{+}$ defined by $\varphi(g \Gamma)=\|\rho(g \gamma) v\|^{2}$, where $\gamma \in \Gamma$ is any element such that $\rho(g \gamma) v \in B_{\delta\left(s_{1}\right)}$, (such a $\gamma$ exists and the value is independent of which one we choose). The crucial point is that if $\left(u_{t}\right)_{t \in \mathbf{R}}$ is as in the theorem then $\varphi\left(u_{i} g \Gamma\right)$, which is defined for $t$ in various intervals, is a polynomial function in $t$ over each of the intervals and the degree of the polynomial is bounded independently of $g$ and the interval. This follows from the following two lemmas.

(2.4) LemmA. Let $x=g \Gamma \in G / \Gamma$, and $0<s<s_{1}$. Let $(a, b)$ be an interval in $\mathbb{R}$, where $a \in \mathbb{R}$ but $b$ may possibly be $\infty$, and suppose that $u_{t} x \in X(\sigma, s)$ for all $t \in(a, b)$. Then there exists $\gamma \in \Gamma$ such that

$$
\varphi(u, x)=\|\rho(u, g \gamma) v\|^{2}<\delta\left(s_{1}\right)^{2}
$$

for all $t \in(a, b)$, ( $\gamma$ being independent of $t)$. A similar assertion holds for closed intervals. 
Proof. Since $X\left(\sigma, s_{1}\right)$ contains a neighbourhood of the closure of $X(\sigma, s)$ we have $u_{a} x \in X\left(\sigma, s_{1}\right)$. By corollary 2.3 we can choose $\gamma$ such that

$$
\left\|\rho\left(u_{a} g \gamma\right) v\right\|<\delta\left(s_{1}\right) .
$$

We prove the assertion in the lemma for this $\gamma$. Since $u_{a} x \in X\left(\sigma, s^{\prime}\right)$ if and only if $s<s^{\prime}$, the second part of that corollary implies that in fact $\left\|\rho\left(u_{a} g \gamma\right) v\right\|=\delta(s)$. Now let $t$ be the smallest value $>a$ such that

$$
\|\rho(u, g \gamma)\|=\frac{1}{2}\left(\delta(s)+\delta\left(s_{1}\right)\right)
$$

choose $t=\infty$ if no such finite value exists. Again, by corollary 2.3 this implies that $u_{t} g \Gamma$ does not belong to the closure of $X(\sigma, s)$. Hence $t \geq b$. In particular, $\left\|\rho\left(u_{t} g \gamma\right)\right\|<$ $\delta\left(s_{1}\right)$ for all $t \in(a, b)$. This implies that the lemma is true for open intervals and a similar argument works for closed intervals.

(2.5) Lemma. Let $g \in G$. Then $\|\rho(u, g) v\|^{2}$ is a polynomial in $t$ whose degree is at most twice the dimension of $V$. Further, there exists $C>0$ such that $\|\rho(u, g) v\|^{2}<C$ for all $t>0$ if and only if $\rho(u, g) v=v$ for all $t \in \mathbb{R}$.

Proof. Since $\left(u_{t}\right)$ is a unipotent one-parameter subgroup, $\rho\left(u_{t}\right)$ is also a one-parameter group of unipotent linear transformations of $V$. By Jordan canonical form the co-ordinates of $\rho\left(u_{t}\right) \rho(g) v$ with respect to any basis of $V$ are polynomials in $t$ of degree at most $\operatorname{dim} V$, the dimension of $V$. Choosing the basis to be an orthonormal basis with respect to the inner product corresponding to the norm $\|\cdot\|$ we see that $\|\rho(u, g) v\|^{2}$ is a polynomial in $t$ of degree at most $2 \operatorname{dim} V$. The second part follows from the fact that the polynomials, corresponding to the coordinates, cannot be bounded over the whole of $\mathbb{R}^{+}$unless they are constant.

The next result shows what distinguishes the unbounded orbits in $G / \Gamma$.

(2.6) Corollary. Let $x=g \Gamma \in G / \Gamma$ be such that the $\left(u_{i}\right)$-orbit of $x$ is not bounded in $G / \Gamma$. Then for any $0<s<s_{1}$ and $a \in \mathbb{R}$ the set

$$
E=\left\{t>a \mid u_{t} x \in X(\sigma, s)\right\}
$$

is a proper subset of $(a, \infty)$.

Proof. Suppose $E=(a, \infty)$. By lemma 2.4 there exists $\gamma \in \Gamma$ such that

$$
\varphi\left(u_{t} x\right)=\left\|\rho\left(u_{t} g \gamma\right) v\right\|^{2}<\delta\left(s_{1}\right)^{2} \quad \text { for all } t \in(a, \infty) .
$$

By lemma 2.5 this implies that $\rho\left(u_{t} g \gamma\right) v=v$ for all $t \in \mathbb{R}$. By the definition of $\rho$, firstly this implies that for all $t \in \mathbb{R}, u_{t} g \gamma$ normalizes $L$. But $\sigma^{-1} P \sigma$ is a maximal subgroup of $G$ normalizing $L$ and hence $u_{t} g \gamma$ must belong to $\sigma^{-1} P \sigma$. But then we must also have $\theta(u, g \gamma)=1$ and hence

$$
u, g \gamma \in \sigma^{-1}(K \cap Z) \cdot N \sigma \quad \text { for all } t \in \mathbb{R} .
$$

But in view of assertion (ii) of theorem $1.1, \sigma^{-1}(K \cap Z) \cdot N \sigma \Gamma / \Gamma$ is a compact subset of $G / \Gamma$, which implies that the $\left(u_{i}\right)$-orbit of $x$ is bounded, thus proving the lemma.

In what follows we shall need the following simple result on the behaviour of non-negative polynomials. 
(2.7) LeMmA. Let $n \in \mathbb{N}, c>0$ and $r>1$ be given. Then there exists $\beta \in(0, c)$ such that the following holds: if $f$ is any non-negative polynomial of degree at most $n$ such that $f(0)=c$, and $f(t) \leq c$ for all $t \in[0,1]$ then there exists $t \in(1, r)$ such that $f(t)>\beta$.

Proof. The space of non-negative polynomials of degree at most $n$ which are bounded over a closed interval is a compact space (under the topology of uniform convergence on compact sets). Now suppose that the lemma is false. Then for all large $j \in \mathbb{N}$ there exists a non-negative polynomial $f_{j}$ of degree $\leq n$ such that $f_{j}(0)=c$ and $f_{j}(t) \leq c$ for all $t \in[0,1]$, and $f_{j}(t) \leq 1 / j$ for all $t \in(1, r)$. By compactness, there exists a polynomial $f$ which is a limit point of $\left\{f_{j}\right\}$. But then we must have $f(0)=c$ and also $f(t)=0$ for all $t \in(1, r)$. This is a contradiction.

\section{Proof of proposition 1.2}

In the proof we shall use the following constants. Let $n \in \mathbb{N}$ be such that $n / 2$ is the dimension of $V$. Let $S \in\left(0, s_{1}\right)$ and $c=\delta(S)^{2}$. Let $r>1$ be such that $n(r-1)<\varepsilon$, where $\varepsilon>0$ is as in the statement of the proposition. Let $\beta \in(0, c)$ be such that the contention of lemma 2.7 holds for the above values of $n, c$ and $r$. Finally let $s \in(0, S)$ be such that $\beta=\delta(s)^{2}$. We shall show that the proposition holds for this value of $s$.

Let $x=g \Gamma$ be an arbitrarily chosen element whose $\left(u_{t}\right)$-orbit is unbounded. Put

$$
E_{0}=\left\{t>0 \mid u_{t} x \in X(\sigma, S)\right\}
$$

and

$$
E_{1}=\left\{t>0 \mid u_{t} x \in X(\sigma, s)\right\} .
$$

Then $E_{0}$ and $E_{1}$ are open subsets of $\mathbb{R}^{+}$. Further in view of corollary 2.6 they are unions of bounded open intervals (possibly empty-but that does not make any difference). Let $(a, b)$ be one of the connected components of $E_{0}$ such that $a>0$. We shall prove that for all $T \in[a, b]$

$$
l\left(E_{1} \cap[a, T]\right) \leq \varepsilon(T-a)=l\left(E_{0} \cap[a, T]\right)
$$

where $l$ is the usual Lebesgue measure on $\mathbb{R}$.

Let $\varphi: X\left(\sigma, s_{1}\right) \rightarrow \mathbb{R}^{+}$be the function defined following corollary 2.3. By lemma 2.4 there exists $\gamma \in \Gamma$ such that for all $t \in[a, b]$

$$
\varphi(u, g \Gamma)=\|\rho(u, g \gamma) v\|^{2} \text {. }
$$

Arguing as in the proof of lemma 2.4 we see that

$$
\varphi\left(u_{a} x\right)=\varphi\left(u_{b} x\right)=\delta(S)^{2}=c .
$$

Similarly if $t \in[a, b]$ is an endpoint of any connected component of $E_{1} \cap[a, b]$ then

$$
\|\rho(u, g \gamma) v\|^{2}=\beta=\delta(s)^{2} .
$$

Since the left hand side of (3.2) is a polynomial (cf. lemma 2.5) in $t$ of degree at most $\boldsymbol{n}$ (twice the dimension of $\boldsymbol{V}$ ) there are at most $\boldsymbol{n}$ such endpoints (and fewer intervals). Note also that in view of the above, neither $a$ nor $b$ is such an endpoint. Let $t_{1}, t_{2}, \ldots, t_{p} \in(a, b)$ where $t_{1}<t_{2} \cdots<t_{p}$ is such that each $t_{i}$ is an endpoint of a connected component of $E_{1} \cap[a, b]$ but not a common endpoint of two such 
successive intervals. Then $p$ is an even number $\leq n$ and

$$
E_{1} \cap[a, b]=\bigcup_{i=1}^{p / 2}\left(t_{2 i-1}, t_{2 i}\right)-J
$$

where $J$ is a finite subset of $[a, b]$, each element of which is a common endpoint of successive intervals in $E_{1}$ and hence satisfies (3.2).

For each of $i=1,2, \ldots, p / 2$ define a non-negative polynomial $f_{i}$ by

$$
f_{i}(t)=\left\|\rho\left(u_{v_{i}(t)} g \gamma\right) v\right\|^{2},
$$

where $\nu_{i}$ is the affine transformation of $\mathbb{R}$ defined by

$$
\nu_{i}(t)=\left(t_{2 i-1}-a\right) t+a \text {. }
$$

Let $i$ be an index between 1 and $p / 2$. Then $f_{i}$ is a non-negative polynomial of degree $\leq n$ such that $f_{i}(0)=c$ and $f_{i}(t) \leq c$ for all $t \in[0,1]$. Therefore by lemma 2.7 there exists $t \in(1, r)$ such that $f_{i}(t)>\beta$. On the other hand by (3.3) we find that for all $t \in\left(1, \nu_{i}^{-1}\left(t_{2 i}\right)\right), \nu_{i}(t) \in E_{1} \cup J$ and consequently $f_{i}(t) \leq \beta$. Therefore $\nu_{i}^{-1}\left(t_{2 i}\right)<r$. Thus $t_{2 i}<\nu_{i}(r)=\left(t_{2 i-1}-a\right) r+a$. Equivalently

$$
\left(t_{2 i}-t_{2 i-1}\right)<\left(t_{2 i-1}-a\right)(r-1), \quad i=1,2, \ldots, p / 2 .
$$

We shall now prove (3.1). For simplicity of presentation let us also put $t_{0}=a$ and $t_{p+1}=b$. Let $T \in[a, b]$ and let $j$ be the largest integer $\leq p / 2$ such that $T \geq t_{2 j}$. Let $\chi$ be the characteristic function on $[a, b]$ of the half open interval $\left(t_{2 j+1}, b\right]$. Then by (3.3) and (3.4) we have

$$
\begin{aligned}
l\left(E_{1} \cap[a, T]\right) & =\sum_{i=1}^{j}\left(t_{2 i}-t_{2 i-1}\right)+\chi(T)\left(T-t_{2 j+1}\right) \\
& \leq \sum_{i=1}^{j}\left(t_{2 i-1}-a\right)(r-1)+\chi(T)\left(t_{2 j+1}-a\right)(r-1) \\
& \leq j(T-a)(r-1)+\chi(T)(T-a)(r-1) \\
& =(T-a)(r-1)(j+\chi(T)) \\
& \leq \varepsilon(T-a),
\end{aligned}
$$

since $j+\chi(T) \leq p \leq n$ and $n(r-1)<\varepsilon$. This proves (3.1).

Now let $T>0$ be arbitrary. Let $I$ be the family of connected components $I$ of $E_{0}$ such that $I \subset(0, T)$ and 0 or $T$ is not an endpoint of $I$. Let $I_{0}=\left[0, b_{0}\right)$ be the connected component of $E_{0} \cup\{0\}$ containing 0 . Let $I_{1}$ be the connected component $E_{0} \cup\{T\}$ containing $T$. Evidently for $T>b_{0},\{I \in \mathscr{I}\}, I_{0}$ and $I_{1} \cap[0, T]$ together form a partition of $E_{0} \cap[0, T]$. We then have for $T>b_{0}$,

$$
\begin{aligned}
l\left(E_{1} \cap[0, T]\right)= & \sum_{l \in \mathscr{S}} l\left(E_{1} \cap I\right)+l\left(E_{1} \cap I_{0}\right)+l\left(E_{1} \cap I_{1} \cap[0, T]\right) \\
& \leq \varepsilon \sum_{l \in \mathscr{S}} l\left(E_{0} \cap I\right)+b_{0}+\varepsilon l\left(E_{0} \cap I_{1} \cap[0, T]\right) \\
& \leq \varepsilon l\left(E_{0} \cap[0, T]\right)+b_{0} \\
& \leq \varepsilon T+b_{0} .
\end{aligned}
$$


Observe that if $\chi_{s}$ is the characteristic function of $X(\sigma, s)$ on $G / \Gamma$ then for any $T$,

$$
\int_{0}^{T} \chi_{s}\left(u_{t} x\right) d t=l\left(E_{1} \cap[0, T]\right) \text {. }
$$

Therefore, noting that $b_{0}$ is a constant (independent of $T$ ) we deduce from (3.5) that

$$
\underset{T \rightarrow \infty}{\limsup } \frac{1}{T} \int_{0}^{T} \chi_{s}(u, x) d t=\limsup _{T \rightarrow \infty} \frac{1}{T} l\left(E_{1} \cap[0, T]\right) \leq \varepsilon
$$

This completes the proof of proposition 1.2 and in turn of theorem 0.2 .

\section{Conclusions}

Combining theorem 0.2 with the results of [2] we can deduce the following theorems.

(4.1) Theorem. Let $G$ be a connected Lie group and $\Gamma$ be a lattice in $G$. Let $\left(u_{t}\right)$ be a unipotent one-parameter subgroup of $G$. Then for any $x \in G / \Gamma$ there exists a compact subset $C$ such that

$$
\liminf _{T \rightarrow \infty} \frac{1}{T} \int_{0}^{T} \chi_{C}(u, x) d t>0,
$$

where $\chi_{c}$ denotes the characteristic function of $C$ on $G / \Gamma$.

Proof. There exists a unique minimum closed normal subgroup $M$ of $G$ such that $G / M$ is a semisimple Lie group with trivial centre and without compact factors. Further $M \Gamma$ is a closed subgroup and the natural quotient map $G / \Gamma \rightarrow G / M \Gamma$ is proper (cf. [1, lemma 9.1]). Therefore replacing $G$ by $G / M$ if necessary (and $\Gamma$ by $M \Gamma / M$ ) we may assume $G$ itself to be a semisimple Lie group with trivial centre and without compact factors. Then there exist (closed) normal subgroups $G_{1}, G_{2}, \ldots, G_{r}$ of $G$ such that $G=G_{1} \cdot G_{2} \ldots \cdot G_{r}$ (direct product) and for each $i=1,2, \ldots, r, \Gamma_{i}=G_{i} \cap \Gamma$ is an irreducible lattice in $G_{i}$ (cf. [7]). Evidently $\Gamma^{\prime}=\Gamma_{1} \Gamma_{2} \ldots \Gamma_{r}$ is a subgroup of finite index in $\Gamma$ and hence in proving the theorem we may without loss of generality assume it to be the whole. The theorem would therefore follow if we prove it for each of $G_{i}$ and $\Gamma_{i}$ in the place of $G$ and $\Gamma$. In other words we may assume $\Gamma$ to be an ireducible lattice.

The contention of the theorem is obvious if $G / \Gamma$ is compact and on the other hand it follows from theorem 0.2 if the $\mathbb{R}$-rank of $G$ is 1 . If neither of these holds then by Margulis's arithmeticity theorem $\Gamma$ is an arithmetic lattice in $G$ (in the sense of $[7, \S 10])$ and therefore the theorem follows from theorem 2.10 of [2] and proposition 10.15 of [7].

(4.2) Remark. An assertion analogous to theorem 4.1 for a cyclic subgroup generated by a unipotent element can easily be deduced from the former.

(4.3) TheOREM. Let $G$ be a Lie group and $\Gamma$ be a lattice in $G$. Let $U$ be a subgroup of $G$ consisting only of unipotent elements. Let $\pi$ be a locally finite $U$-invariant measure on $G / \Gamma$. Then there exist measurable $U$-invariant subsets $X_{i}, i \in \mathbb{N}$, such that $\pi\left(X_{i}\right)<\infty$ for all $i$ and $G / \Gamma=\bigcup_{i=1}^{\infty} X_{i}$. In particular every locally finite ergodic U-invariant measure is finite.

Proof. This can be deduced from theorem 4.1 and the individual ergodic theorem following almost verbatim the proof of theorem 3.3 in [2]. We omit the details. 


\section{REFERENCES}

[1] S. G. Dani. Invariant measures of horospherical flows on non-compact homogeneous spaces. Invent. Math. 47 (1978), 101-138.

[2] S. G. Dani. On invariant measures, minimal sets and a lemma of Margulis. Invent. Math. 51 (1979), 239-260.

[3] S. G. Dani. Invariant measures and minimal sets of horospherical flows. Invent. Math. 64 (1981), 357-385.

[4] S. G. Dani \& John Smillie. Uniform distribution of horocycle orbits for Fuchsian groups. Duke Math. J. 51 (1984), 185-194.

[5] H. Garland \& M. S. Raghunathan. Fundamental domains for lattices in R-rank 1 semisimple Lie groups. Ann. of Math. 92 (1970), 279-326.

[6] G. A. Margulis. On the action of unipotent groups in the space of lattices. Proc. of the Summer School on Group Representations, Budapest, Bolyai Janos Math. Soc., pp. 365-370, 1971.

[7] M. S. Raghunathan. Discrete Subgroups of Lie Groups. Springer-Verlag: Berlin-Heidelberg-New York (1972). 